\title{
The clinical evaluation of IIA balloon occlusion in caesarean delivery for patients with PAS: a retrospective study
}

Ling Hong ${ }^{1 \dagger}$, Aner Chen ${ }^{1 \dagger}$, Jinliang Chen ${ }^{2}$, Xiuxiu Li ${ }^{3}$, Wenming Zhuang ${ }^{1}$, Yijing Shen ${ }^{3}$, Qiaohong Dai ${ }^{1}$ and Li Zhang ${ }^{3^{*}}$

\begin{abstract}
Objective: The aim of this study is the clinical evaluation of IIA balloon occlusion in the caesarean delivery in patients with a diagnosis of placenta accreta spectrum.

Background: High incidence of cesarean section leads to the increasing incidence of placenta accreta spectrum (PAS), which contributes to serious consequences such as severe obstetric postpartum hemorrhage or even maternal mortality.

Methods: Fifty-eight patients with a diagnosis of PAS were retrospectively reviewed. The balloon group consisted of 23 patients, who underwent a caesarean delivery with internal iliac artery occlusion. 35 patients were in the control group, who had a standard caesarean delivery. The primary outcomes were estimated blood loss (EBL). The secondary outcomes were cesarean hysterectomy, blood transferring volume, operating time, intraoperative hemostatic approaches, surgical complications, balloon catheter-related complications, length of maternal stay, cost of hospitalization, and neonatal outcomes.

Results: No difference was observed in estimated blood loss (EBL), blood transferring percentages and volume, additional measures to secure hemostasis, surgical complications, hospital stay postoperatively and newborn outcomes. More than $40 \%$ of the balloon group underwent hysterectomy because of uncontrollable postpartum bleeding (10 [43.48\%] vs. 11 [31.43\%], $P=0.350)$. Complications related to occlusion of IIA did not occur. The duration of the surgery of the balloon group was significantly longer than that of the control group (123.52 min \pm 74.76 versus $89.17 \pm 48.68, P=0.038$ ), and the total hospitalization cost was also significantly higher than that of the control group (45116.67 \pm 9358.67 yuan versus $30615.41 \pm 11587.44$ yuan, $P=0.000$ ).

Conclusion: It does not permit to draw final conclusions for us on the effectiveness of the balloons IIA given the heterogeneity of selection of cases undergoing the procedures in the retrospective design. However, it is possible that IIA balloon occlusion may contribute to limiting intraoperative blood loss in more severe cases, particularly those undergoing peripartum hysterectomy.
\end{abstract}

Keywords: Placenta accreta spectrum, Placenta accrete, IIA balloon occlusion

\section{*Correspondence: zhangli00501465@outlook.com}

'Ling Hong and Aner Chen are contributed equally to this work and should be considered co-first authors.

${ }^{3}$ Department of science and education, Ningbo women and children's hospital, 339 liuting street, Ningbo city, Zhejiang province, China Full list of author information is available at the end of the article

\section{Background}

The term placenta accreta spectrum disorder (PASD) is used to describe the morbidly adherent placenta, which includes placenta accreta, increta and percreta [1]. In past decades, women increasingly preferred to deliver by 
cesarean section (CS), leading to the increasing incidence of placenta accreta spectrum (PAS) [2]. It is reported with an incidence of 1 per 533 pregnancies [3]. One of the main and deadliest complications of PAS is massive bleeding. The average blood loss in these cases has been estimated to range between 2 and 3 liters [4]. Additionally, the morbidly adherent placenta is the most common indication for peripartum hysterectomy, which is associated with high rates of morbidity and mortality. Therefore, strategies to prevent and treat bleeding are are therefore critical. Various approaches have been employed in controlling intraoperative blood loss in cases of abnormal placentation, including inserting uterine tamponade balloons, applying uterine gauze packing, placing uterine brace or isthmic compression sutures, and performing a hysterectomy.

The arterial occlusion balloon has been used in PAS recently, and the role of internal iliac artery (IIA) balloon occlusion to improve hemorrhagic outcomes in women with placenta accreta has been evaluated in many studies, some of which showed benefit [5-9], but others failed to demonstrate any [10-12]. The aim of this study is the clinical evaluation of IIA balloon occlusion in the caesarean delivery in patients with a diagnosis of placenta accreta spectrum.

\section{Methods}

Fifty-eight patients with a diagnosis of PAS underwent caesarean delivery between January 2015 and June 2018 at Ningbo Women and Children's Hospital were retrospectively reviewed in this study. The Institutional Review Board of Ningbo Women and Children's approved this study, and the need for informed consent was waived because of the retrospective nature of this study.
Electronic medical records were reviewed to collect patient demographics, obstetrical history, operative reports, and clinical notes. The inclusion criteria were a diagnosis of PAS and having caesarean sections performed by the same experienced surgeon. For all women included in this study, a diagnosis of PAS had been made in prenatal imaging with either ultrasound and/or magnetic resonance imaging (Fig. 1) and abnormal placental status was confirmed clinically [1], or histopathologically, after delivery. The following patients were excluded: those who had a history of gynecological cancers or other solid cancers, hematological malignancies, gynecological surgeries (such as myomectomy, cervicoplasty and uterine rupture repair); those severe platelet disorders; twin pregnancy, those who underwent emergent delivery and cases with missing data. Each clinical case was discussed in multidisciplinary meetings with interventional radiologists, obstetricians, neonatologists, and urologist. The women who had consented to and undergone IIA balloon occlusion were assigned to the balloon group, while others were assigned to the control group. Patients who were not diagnosed antenatal but were diagnosed with placental implantation after delivery may also be included in the control group. Finally, there were 23 patients in the balloon group and 35 patients in the control group. Two women who underwent balloon occlusion but did not end up with a diagnosis of PAS were excluded. Occlusion balloon placement was performed by one of two senior interventional radiologists, who each had more than five years of experience with the similar procedure of uterine artery embolisation for caesarean scar ectopic pregnancy.

Procedure for the management of IIA balloon catheters was as follows. Arteriopuncture of bilateral femoral arteries via the Seldinger technique was performed after local anesthesia; 6- French sheaths were then put in place.
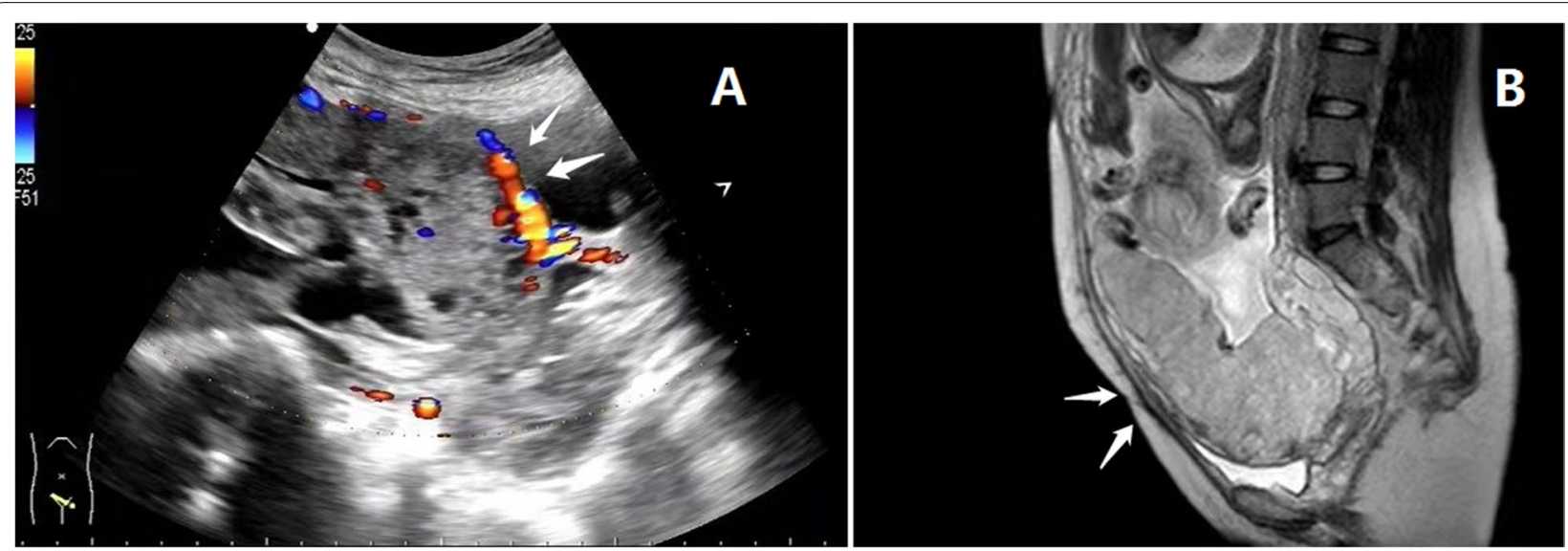

Fig. 1 A Color Doppler images. The arrow indicates evidence of placenta accreta (indistinct boundary of utero-placental interface); B MRI. Placenta accreta in the lower uterine segment (indicated by an arrow) 
Next, selective contralateral IIA catheterization was performed with fluoroscopy guidance, and a PTA catheter (Abbott, USA) was inserted into the IIA. The balloon (Abbott, USA), which was approximately $8-10 \mathrm{~mm}$ in diameter, was exchanged by using a 0.035 - inch superhard exchange guide wire and then positioned in the IIA with the head pointing to the branch of the anterior IIA, as confirmed by angiography (Fig. 2). After the insertion, The balloon was briefly inflated, and contrast injected to verify occlusion of the artery. Once positioning was satisfactory, the balloon catheter and its sheath were fixed on the skin. Patients were then transferred to the operating room for cesarean delivery. After the baby was delivered, the occlusion balloons were inflated immediately. The placenta was then delivered and cesarean delivery was continued in the usual manner. Balloons were inflated for approximately 30 minutes intraoperatively The balloon catheters were deflated after hemostasis was achieved, and 1 sheath was left in place for 12 hours to allow for resuscitation and emergency embolization if needed. The balloon was inflated or deflated by an interventional radiologist during the whole CS. Perioperative management was similar in the two groups except for catheter

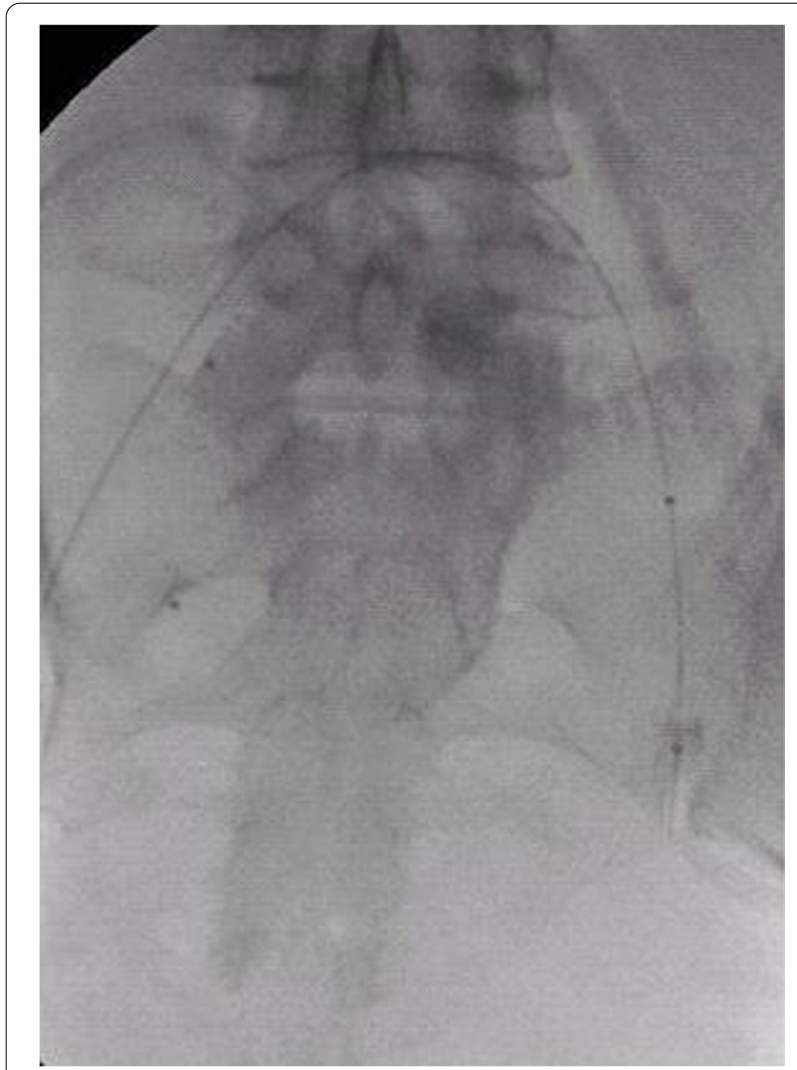

Fig. 2 Fluoroscopy showing the balloon catheters positioned in the main lumen of the internal iliac arteries bilaterally placement and balloon occlusion of bilateral internal iliac arteries.

The primary outcomes were estimated blood loss (EBL). The secondary outcomes were as follows: cesarean hysterectomy, blood transferring volume, operating time, intraoperative hemostatic approaches ( such as intrauterine tamponade, compression suturing, uterine artery ligation), surgical complications, balloon catheter-related complications (including puncture site hematoma, thrombosis, embolic events, and vascular rupture), length of maternal stay, cost of hospitalization, and neonatal outcomes. Statistics Statistical analysis was performed using SPSS 20.0 software. Data were presented as mean $\pm \mathrm{SD}$, median (range) or count (percentage). Comparison between 2 groups was determined by $\mathrm{t}$ test, Wilcoxon rank sum test or chi-square test. $p<0.05$ was considered significant.

\section{Result}

Fifty-eight women were included. Twenty- three women were in the balloon group and thirty-five women were in the control group. Baseline characteristics are summarized in Table 1. There was no significant difference between the two groups with respect to patient age at delivery, gestational age, gravidity, parity, type of pregnancy, number of previous uterine surgeries and abdominal surgeries, number of previous cesarean deliveries, types of placenta accretion and hemoglobin before cesarean section.

The intraoperative and postoperative outcomes for the two groups are summarized in Tables 2 and 3. The median EBL was $2500 \mathrm{ml}$ (range, $1750-5750 \mathrm{ml}$ ) in the balloon group versus $2000 \mathrm{ml}$ (range, 1500-3350ml) in the control group ( $p=0.171)$. There was no statistically significant difference observed between the groups with intraoperative hemorrhage volumes of $2500 \mathrm{~mL}$ and above $(p=0.421)$. More than $40 \%$ of the balloon group underwent hysterectomy because of uncontrollable postpartum bleeding (10[43.48\%] vs. $11[31.43 \%], p=0.350)$. The need for additional measures to secure hemostasis similarly showed no difference between the groups (Table 3 ).

Twenty-one women in the balloon group and all women in the control group received blood transfusions. No significant differences were noted both in the percentages and volumes of autologous blood transfusion, red blood cell transfusion, fresh frozen plasma transfusion and cryoprecipitate transfusion between the two groups. There was no significant difference in the $\mathrm{Hb}$ decrease between the two groups (1.3 (range, -1.5-1.7) vs 1.35 (range, 0.1-2.45), $p=0.266$ ).

The duration of the surgery of the balloon group was significantly longer than that of the control group (123.52 $\min \pm 74.76$ versus $89.17 \pm 48.68, p=0.038$ ), and the total 
Table 1 Demographic characteristics and incidence of different forms of placenta accretion in the two study groups

\begin{tabular}{llll}
\hline Characteristic/Outcome & Balloon Group $(\boldsymbol{n}=\mathbf{2 3})$ & Control group $(\boldsymbol{n}=\mathbf{3 5})$ & $\boldsymbol{p}$-value \\
\hline Mean age, years & $33.00 \pm 3.77$ & $33.37 \pm 4.44$ & 0.742 \\
gestational age, weeks & $34.86 \pm 1.05$ & $35.47 \pm 1.20$ & 0.113 \\
Gravidity & $3.70 \pm 1.11$ & $3.91 \pm 1.29$ & 0.508 \\
Parity & $2.13 \pm 0.55$ & $2.14 \pm 0.43$ & 0.924 \\
Number of previous uterine surgeries & $1.52 \pm 1.47$ & $1.74 \pm 1.17$ & 0.528 \\
Number of previous cesarean deliveries & $1.09 \pm 0.29$ & $1.14 \pm 0.36$ & 0.531 \\
Hemoglobin before cesarean section, g/dl & $11.23 \pm 1.33$ & $11.19 \pm 1.25$ & 0.914 \\
Deepth of Placenta percreta & & & \\
$\quad$ Accreta & 4 & 11 & 0.232 \\
Increta & $9(56.52 \%)$ & $16(77.14 \%)$ & 0.620 \\
Percreta & $10(43.48 \%)$ & $8(22.86 \%)$ & 0.097 \\
\hline
\end{tabular}

Table 2 Blood loss and transfusion requirements between the two study groups

\begin{tabular}{|c|c|c|c|}
\hline Parameters & Balloon Group $(n=23)$ & Control group $(n=35)$ & $p$-value \\
\hline Estimated blood loss, $\mathrm{ml}^{\mathrm{a}}$ & $2500(1750-5750)$ & $2000(1500-3350)$ & 0.226 \\
\hline Blood loss $\geq 2500 \mathrm{ml}, \mathrm{n}(\%)$ & $13(56.52 \%)$ & $16(45.71 \%)$ & 0.421 \\
\hline Transfusion, n(\%) & $21(91.30 \%)$ & $35(100 \%)$ & 0.076 \\
\hline Autologous blood transfusion, n(\%) & $20(86.96 \%)$ & $34(97.14 \%)$ & 0.134 \\
\hline Autologous blood transfusion, $\mathrm{ml}^{\mathrm{a}}$ & $600(335-1050)$ & $500(278.5-745.5)$ & 0.347 \\
\hline RBC transfusion, $\mathrm{n}(\%)$ & $12(52.17 \%)$ & $17(48.57 \%)$ & 0.788 \\
\hline RBC, units $^{\mathrm{a}}$ & $3(0-6)$ & $0(0-4.5)$ & 0.486 \\
\hline FFP transfusion, $\mathrm{n}(\%)$ & $18(78.26 \%)$ & $20(57.14 \%)$ & 0.098 \\
\hline FFP, units ${ }^{\mathrm{a}}$ & 1080(555-1200) & $500(0-1160)$ & 1.000 \\
\hline Cryoprecipitate transfusion, n(\%) & $12(52.17 \%)$ & $10(28.57 \%)$ & 0.070 \\
\hline 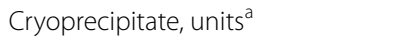 & $5(0-10)$ & $0(0-9.75)$ & 0.343 \\
\hline
\end{tabular}

RBC Red blood cells, FFP Fresh frozen plasma

${ }^{a}$ Data were presented as median (range). Comparison between 2 groups was determined by Wilcoxon rank sum test

hospitalization cost was also significantly higher than that of the control group (45116.67 \pm 9358.67 yuan versus $30615.41 \pm 11587.44$ yuan, $p=0.000$ ). There was no significant difference in surgical complications, postoperative hospital stay, newborn weight, Apgar scores at 1 and 5 minutes between the two groups. No still births or maternal death was observed in either group. No balloon related complication occurred in the balloon group.

\section{Discussion}

This study showed that balloon occlusion of the IIA in patients with PAS did not reduce the hysterectomy rate during a cesarean section, nor did it reduce blood loss and blood transfusion, but it prolonged the duration of the surgery and increased the total cost.

Although advances in obstetric care have led to a substantial improvement in pregnancy outcomes, the death rate from PAS remains as high as $7 \%$, largely due to massive hemorrhage [13, 14], which may then lead to disseminated intravascular coagulation, fluid overload, acute respiratory distress syndrome and infection. It seems logical that occlusion of the internal iliac arteries with prophylactically- placed balloon catheters would be a more effective treatment option, but reported results are controversial. Some retrospective studies [6-9] reported that intraoperative IIA balloon occlusion had benefits in reducing blood loss and the amount of blood transfusion. Some previous systematic reviews also reported that intraoperative IIA balloon occlusion had benefits in reducing blood loss, the volumes of blood transfusion and even the percentages of cesarean hysterectomy in women with accrete [5, 15]. Nicholson et al. Also reported that patients with IIA balloon occlusion had a decreased rate of hysterectomy compared to those without it [16]. Recently, Yao FAN and Soo BuemCho 's two randomized controlled studies also agree with this view $[15,17]$. However, randomized controlled studies $[10,18]$ 
Table 3 Surgical details and postoperative outcomes between the two study groups

\begin{tabular}{|c|c|c|c|}
\hline Parameters & Balloon Group $(n=23)$ & Control group $(n=35)$ & $P$-value \\
\hline Cesarean hysterectomy, n(\%) & $10(43.48 \%)$ & $11(31.43 \%)$ & 0.350 \\
\hline Additional measures to secure hemostasis, n(\%) & $15(65.22 \%)$ & $27(77.14 \%)$ & 0.320 \\
\hline lodoform gauze packing of the uterine cavity, n(\%) & $14(60.87 \%)$ & $26(74.29 \%)$ & 0.280 \\
\hline Balloon tamponade, $\mathrm{n}(\%)$ & $8(34.78 \%)$ & $20(57.14 \%)$ & 0.096 \\
\hline Uterine artery ligation, n(\%) & $2(8.70 \%)$ & $2(5.71 \%)$ & 0.661 \\
\hline uterine artery embolization, n(\%) & $6(26.09 \%)$ & 12(34.29\%) & 0.509 \\
\hline Partial excision of the invaded uterine wall, $n(\%)$ & 3 & 2 & 0.303 \\
\hline Duration of the surgery, min* & $123.52 \pm 74.76$ & $89.17 \pm 48.68$ & 0.038 \\
\hline Hospital stay postoperatively, days & $7.96 \pm 2.14$ & $7.74 \pm 1.90$ & 0.692 \\
\hline Hospitalization cost, yuan* & $45116.67 \pm 9358.67$ & $30615.41 \pm 11587.44$ & 0.000 \\
\hline Surgical complications, n(\%) & $14(60.87 \%)$ & $22(62.86 \%)$ & 0.879 \\
\hline Fever, n(\%) & $11(47.83 \%)$ & $20(57.14 \%)$ & 0.487 \\
\hline $\mathrm{DIC}, \mathrm{n}(\%)$ & $2(8.70 \%)$ & $4(11.43 \%)$ & 0.738 \\
\hline Pneumonedema, n(\%) & 0 & $1(2.86 \%)$ & 0.414 \\
\hline Bladder injury, n(\%) & $3(13.04 \%)$ & $1(2.86 \%)$ & 0.134 \\
\hline Relaparotomy, n(\%) & $2(8.70 \%)$ & $3(8.57 \%)$ & 0.987 \\
\hline Maternal death & 0 & 0 & - \\
\hline \multicolumn{4}{|l|}{ Balloon related complications } \\
\hline Hematoma puncture & 0 & 0 & - \\
\hline Pseudoaneurisms & 0 & 0 & - \\
\hline Vein thrombosis & 0 & 0 & - \\
\hline \multicolumn{4}{|l|}{ Neonatal outcome } \\
\hline Neonatal birth weight, $g$ & $2540 \pm 480$ & $2740 \pm 360$ & 0.074 \\
\hline Apgar score of $1 \mathrm{~min}$ & $8.70 \pm 0.56$ & $8.46 \pm 1.12$ & 0.349 \\
\hline Apgar score of $5 \mathrm{~min}$ & $9.78 \pm 0.42$ & $9.69 \pm 0.93$ & 0.642 \\
\hline Neonatal mortality & 0 & 0 & - \\
\hline
\end{tabular}

DIC disseminated intravascular coagulation

${ }^{*} P<0.05$

and several case control studies [11, 12, 19] did not find any benefit. Our findings accord with the previous randomized controlled trial conducted by Meng Chen et al [18].

In our study, all patients are operated by the same surgeon, who has more than 20 years of experience and performs nearly 1,000 cesarean sections a year. They also received the same standard peripartum care apart from the insertion of iliac artery balloons which also eliminate the surgical procedural heterogeneity between surgeons and teams. This is the most significant advantage of our research, which has not been reported in other studies.

In our study, the two groups had similar blood loss, it might be possible that balloons contributed to limit blood loss of more severe cases (more percreta and increta in the IIA balloon group and more accreta in the control group, although not statistically significant), so we cannot exclude a role of the balloons in reducing blood loss in more severe cases. Given difficulties of prenatal diagnosis of PAS, should we consider positioning balloons only in suspected percretas? It might be worth digging into. On the other hand, collateral circulation of the ovarian artery and balloon displacement may also one of the reasons why occlusion of the internal iliac arteries failed to reduce hemorrhage [20, 21].

In our study, the transfusion rate was $91.3 \%$ in the balloon group and $100 \%$ in the control group, which was much higher than the $50 \%$ or so reported in the literature [22]. In our hospital, autologous blood transfusion is one of the necessary means to reduce the bleeding during the operation of pregnant women with placenta accreta spectrum. Therefore, nearly every pregnant woman in our study underwent autologous blood transfusion during a cesarean section, which may be the reason for the increased transfusion rate in our study.

Our results show that the duration of surgery in the balloon group is significantly longer than in the control group, which should be interpreted with caution. Pregnant women in the balloon group needed an 
interventional surgeon to remove the balloon after surgery, whereas women in the control group did not.

This study has several limitations, the most important of which was that the decision of balloon positioning was highly dependent on physician consultation with patients, and therefore there is the suspicion of a selection bias (balloon positioned in more severe cases). Then, we consider all the PAS equally, not differentiating grade 1,2 or 3 . In the study, blood loss was estimated, and transfusion was performed based on surgeon preference, which may have varied based on practice.

\section{Conclusion}

It does not permit to draw final conclusions for us on the effectiveness of the balloons IIA given the heterogeneity of selection of cases undergoing the procedures in the retrospective design. However, it is possible that IIA balloon occlusion may contribute to limiting intraoperative blood loss in more severe cases, particularly those undergoing peripartum hysterectomy.

\begin{abstract}
Abbreviations
PASD: Placenta accreta spectrum disorder; PAS: Placenta accreta spectrum; IIA: Internal iliac artery; EBL: Estimated blood loss; RBC: Red blood cells; FFP. Fresh frozen plasma; Hb: Haemoglobin concentration; DIC: Disseminated intravascular coagulation.
\end{abstract}

\section{Acknowledgements}

Not applicable.

\section{Authors' contributions}

LH: data collection and analysis; primary author of manuscript. AC: evaluation design; Surgeon of cesarean section; JC: Imaging technical guidance, image collection. XL, ,YS,QD: data analysis and interpretation; manuscript preparation and review. WZ: obtaining funding and ethics approval. LZ: leads all aspects of this study including study concept and design; obtaining funding and ethics approval; supervision of data collection and implementation; and manuscript supervision and revision for critical intellectual input. All authors have read and approved the manuscript.

\section{Funding}

The study was supported by the grant from Health Commission of Zhejiang Province 2020PY024, Ningbo Science and Technology Bureau 2019C50090 and Ningbo Municipal Health Commission, PPXK2018-06

\section{Availability of data and materials}

The research data used to support the findings of this study were supplied by Ms. Zhang under license and so cannot be made freely available. Requests for access to these data should be made to Ms. Zhang (Email:zhangli00501465@ outlook.com).

\section{Declarations}

\section{Ethics approval and consent to participate}

The Institutional Review Board of Ningbo Women and Children's approved this study. The need for informed consent was waived by the Institutional Review Board of Ningbo Women and Children's because of retrospective nature of this study. (EC2019-043). Research was conducted according to the ethical standard of Helsinki declaration and that data of patients were stored anonymized in a dedicated database. All methods were carried out in accordance with relevant guidelines and regulations.
Consent for publication

Not applicable for this publication.

\section{Competing interests}

We declare that we don't have competing interests with publication of this article.

\section{Author details}

${ }^{1}$ Department of obstetrics and gynecology, Ningbo women and children's hospital, Ningbo, China. ${ }^{2}$ Radiology department, Ningbo women and children's hospital, Ningbo, China. ${ }^{3}$ Department of science and education, Ningbo women and children's hospital, 339 liuting street, Ningbo city, Zhejiang province, China.

Received: 13 September 2021 Accepted: 27 January 2022

Published online: 05 February 2022

\section{References}

1. Collins SL, Alemdar B, van Beekhuizen HJ, Bertholdt C, Braun T, Calda P, et al. International Society for Abnormally Invasive Placenta (IS-AIP). Evidence-based guidelines for the management of abnormally invasive placenta: recommendations from the International Society for Abnormally Invasive Placenta. Am J Obstet Gynecol. 2019;220(6):511-26. https://doi. org/10.1016/j.ajog.2019.02.054 Epub 2019 Mar 5. PMID: 30849356.

2. Klar M, Michels KB. Cesarean section and placental disorders in subsequent pregnanciesea meta-analysis. J Perinat Med. 2014;42:571-83.

3. Fan D, Song W, Wang W, Xin L, Tian G, Liu L, et al. Zhengping Liu; Prevalence of placenta previa among deliveries in Mainland China: A PRISMA-compliant systematic review and meta-analysis. Medicine. 2016:95(40):e5107.

4. Shamshirsaz AA, Fox KA, Salmanian B, Diaz-Arrastia CR, Lee W, Baker BW, et al. Maternal morbidity in patients with morbidly adherent placenta treated with and without a standardized multidisciplinary approach. Am J Obstet Gynecol. 2015;212(2):218.e1-218.e2189.

5. Shahin Y, Pang CL. Endovascular interventional modalities for haemorrhage control in abnormal placental implantation deliveries: a systematic review and meta-analysis. Eur Radiol. 2018;28:2713-26.

6. Dai MJ, Jin GX, Lin JH, Zhang Y, Chen YY, Zhang XB. Pre-cesarean prophylactic balloon placement in the internal iliac artery to prevent postpartum hemorrhage among women with pernicious placenta previa. Int J Gynaecol Obstet. 2018;142:315-20.

7. Zhou X'e, Sun X, Wang M, Huang L. Wen Xiong; The effectiveness of prophylactic internal iliac artery balloon occlusion in the treatment of patients with pernicious placenta previa coexisting with placenta accreta. J Matern Fetal Neonatal Med. 2021 Jan;34(1):93-8.

8. McGinnis JM, Simula NK, Joseph KS, Ubhi JS. Internal iliac artery balloon tamponade in placenta accreta: outcomes from the largest tertiary accreta referral centre in British Columbia. J Obstet Gynaecol Can. 2019;41:466-72.

9. Gulino FA, Guardo FD, Zambrotta E, Di Gregorio LM, Miranda A, Capriglione S, et al. Placenta accreta and balloon catheterization: the experience of a single center and an update of latest evidence of literature. Arch Gynecol Obstet. 2018;298:83-8.

10. Salim R, Chulski A, Romano S, Garmi G, Rudin M, Shalev E. Precesarean prophylactic balloon catheters for suspected pla-centa accreta: a randomized controlled trial. Obstet Gynecol. 2015;126:1022-8.

11. Chen M, Lv B, He G, Liu X. Internal iliac artery balloon occlusion during cesarean hysterectomy in women with placenta previa accreta. Int J Gynaecol Obstet. 2019;145:110-5.

12. Feng $\mathrm{S}$, Liao $Z$, Huang $\mathrm{H}$. Effect of prophylactic placement of internal iliac artery balloon catheters on outcomes of women with placenta accreta: an impact study. Anaesthesia. 2017;72:853-8.

13. Stotler B, Padmanabhan A, Devine P, Wright J, Spitalnik SL, Schwartz J. Transfusion requirements in obstetric patients with placenta accreta. Transfusion. 2011;51:2627-33.

14. O'Brien JM, Barton JR, Donaldson ES. The management of placenta percreta: conservative and operative strategies. Am J Obstetr Gynecol. 1996;175:1632-8.

15. Fan $Y$, Gong $X$, Wang $N$, Ke-Tao M, Feng L, Qiao F-Y, et al. Xiao-Yan Xu; A Participant-assigned Interventional Research of Precesarean Internal Iliac Artery Balloon Catheterization for Managing Intraoperative Hemorrhage of Placenta Previa and Placenta Accreta Spectrum Disorders After Cesarean Section. Curr Med Sci. 2021;41(2):336-41. 
16. Patrick J. Nicholson, Owen O'Connor, John Buckley, Liam D Spence, Richard A Greene, David J Tuite; Prophylactic Placement of Internal Iliac Balloons in Patients with Abnormal Placental Implantation: Maternal and Foetal Outcomes. Cardiovasc Int Radiol. 2018;41(10):1488-93.

17. Cho SB, Hong SJ, Lee S, Won JH, Choi HC, Ha JY, et al. Preoperative Prophylactic Balloon-Assisted Occlusion of the Internal Iliac Arteries in the Management of Placenta Increta/Percreta. Medicina (Kaunas, Lithuania). 2020;56(8):368.

18. Chen M, Liu X, You Y, Wang X, Li T, Luo H, et al. Internal iliac artery balloon occlusion for placenta previa and suspected placenta accreta: a randomized controlled trial. Obstetr Gynecol. 2020;135(5):1112-9.

19. Shrivastava V, Nageotte M, Major C, Haydon M, Wing D. Case-control comparison of cesarean hysterectomy with and without prophylactic placement of intravascular balloon catheters for placenta accreta. Am J Obstet Gynecol. 2007;197(402):e1-5.

20. Pelage JP, Le Dref $\mathrm{O}$, Soyer $\mathrm{P}$, et al. Arterial anatomy of the female genital tract: variations and relevance to transcatheter embolization of the uterus. Am J Roentgenol. 1999;172:989-94.

21. Teixidor Vi nas M, Chandraharan E, Moneta MV, Belli AM. The role of interventional radiology in reducing haemorrhage and hysterectomy following caesarean section for morbidly adherent placenta. Clin Radiol. 2014;69:e345-51.

22. Nieto-Calvache AJ, Vergara-Galliadi LM, Rodríguez F, Ordoñez CA, García AF, López MC, et al. A multidisciplinary approach and implementation of a specialized hemorrhage control team improves outcomes for placenta accreta spectrum. J Trauma Acute Care Surg. 2021;90(5):807-16.

\section{Publisher's Note}

Springer Nature remains neutral with regard to jurisdictional claims in published maps and institutional affiliations.

- fast, convenient online submission

- thorough peer review by experienced researchers in your field

- rapid publication on acceptance

- support for research data, including large and complex data types

- gold Open Access which fosters wider collaboration and increased citations

- maximum visibility for your research: over 100M website views per year

At BMC, research is always in progress.

Learn more biomedcentral.com/submissions 